\title{
Relationship between Refractive Index and Molar Concentration of Multi-Component Solutions
}

\author{
Zhu Xingyu $^{1, a}$, Mai Tiancheng ${ }^{2, b}$ and Zhao Zilong ${ }^{2, c}$ \\ ${ }^{1}$ The High School Affiliated to Renmin University, Beijing 100080, China; \\ 2 The High School Affiliated to Renmin University, Beijing 100080, China; \\ ${ }^{3}$ The High School Affiliated to Renmin University, Beijing 100080, China. \\ a jupiter_zhu_007@126.com
}

Keywords: Physical optics; Multi-component solution; Refractive index; Molar concentration; Linear relationship.

\begin{abstract}
Variations in molar densities and solute properties, which together form a solution's basic index, can cause changes in the solution's refractive index. The linear relationship between the ternary system's refractive index solution and each solute's molar concentration can be determined through ternary system solution analysis on light passing through the solution. Experiments were conducted on a CuSO4-NaCl-H2O solution's refractive index in this study. The model utilized here can be further applied to multi-component solutions, as the solution's refractive index has a linear relationship with the properties of each solute. A new refractive index model can be derived accordingly to further enhance the results.
\end{abstract}

\section{Introduction}

The refractive index is a fundamental property of a solution which can vary with temperature, composition, concentration [1], and incident light wavelength [2]. The relationship between the solution concentration and refractive index has many applications such as measuring sea salt concentration [3] and beer quality assessment [4]. Glover et al. [5] investigated the relationship between sucrose solution concentration, mass concentration, and the refractive index to establish an index characterized by the time it takes a beam of light to pass through a unit volume of a given solution. Zhang et al. [6] proposed a linear correlation to a binary solution's refractive index ( $\delta=A n+B$ ) based on Lorentz's electronics theory, Lambert's law, and Beer's law. Zhu et al. [7] experimentally investigated the relationship between a ternary inorganic solution's molar concentration and its refractive index, which is also linear. According to the electron cloud inductor model, Zhang [8]suggested that a solution's refractive index can be expressed as $n=1+\sum\left(\rho_{m} D_{m}\right)_{i}$.

This study was carried out based on the refractive index correlation of Snell's law. The influence of variations in a molar solution concentration on its refractive index was investigated by analyzing light passing through solutions with different molar concentrations. The multi-component solution refractive index varied linearly with each solute's molar concentration under the interaction conditions between solute ions and various volumes after controlling for dissolution.

\section{The Model of Ternary System Solution}

As Snell's law suggests, the refractive index of a medium is the ratio of the incident angle's sine to the refracted angle's sine considering the light incidence from the vacuum into the medium, i.e.:

$$
n=\frac{c}{v}=\frac{\sin \theta_{1}}{\sin \theta_{2}}
$$

Where $c$ is the speed of light in a vacuum, $v$ is the speed of light in the medium, and $\theta_{1}$ and $\theta_{2}$ are the incident and refracted angles, respectively. 


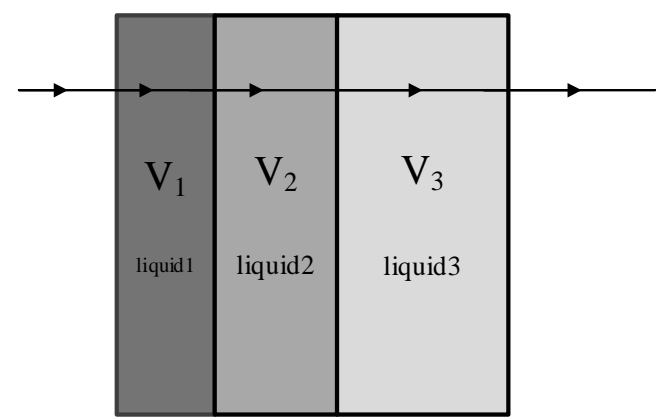

Fig. 1 Schematic diagram of light path through ternary system solution

As shown in Fig. 1, the solution volume is weak because the solid solute dissolves in a solvent. The ternary system solution (two solutes dissolved in a solvent) can also be regarded as a triple-liquid combination with constant molar concentration and refractive index. Liquids 1 and 2 are solutions with constant molar concentration and refractive index which combine withsolutes $X_{1}$ and $X_{2}$, respectively. Liquids 1 and 2 molar concentrations are $\mathrm{kmol} / \mathrm{L}$ and liquid 3 is the solvent (the remaining solvent after removing the solvents combined in liquids 1 and 2) with constant molar concentration and refractive index. Light speed in a vacuum is $c$ and light speed in the solution is $v$, so the light $\operatorname{speedsare} v_{1}, v_{2}$, and $v_{3}$ in the liquids, respectively. The molar concentrations of solutes $X_{1}$ and $X_{2}$ are $p_{1} \mathrm{~mol} / \mathrm{L}$ and $p_{2} \mathrm{~mol} / \mathrm{L}$.

For the ternary system solution unit volume, it is assumed here that the liquid volume with known molar concentration, known refractive index, and unique solute $X_{1}$ is $V_{1}$. The liquid volume with known molar concentration, known refractive index, and unique solute $X_{2}$ is $V_{2}$, and the rest of the solute volume is $V_{3}$. The total distance of light passing through the solution is $L$ and the total time is $T$. The distances in liquid 1, liquid2, and liquid3, are $L_{1}, L_{2}$, and $L_{3}$ and the times are $T_{1}, T_{2}$, and $T_{3}$, respectively.

The qualities of solute $X_{1}$ and $X_{2}$ in the solution are the same as those in liquids 1and2, so:

$$
\begin{gathered}
p_{1}\left(V_{1}+V_{2}+V_{3}\right)=k V_{1} \\
p_{2}\left(V_{1}+V_{2}+V_{3}\right)=k V_{2} \\
p_{1}=k \frac{V_{1}}{V_{1}+V_{2}+V_{3}} \\
p_{2}=k \frac{V_{2}}{V_{1}+V_{2}+V_{3}} \\
L_{1}=L \frac{V_{1}}{V_{1}+V_{2}+V_{3}}=\frac{L p_{1}}{k} \\
L_{2}=\frac{L p_{2}}{k} \\
L_{3}=\frac{L\left(1-p_{1}-p_{2}\right)}{k} \\
t_{1}=\frac{L p_{1}}{k v_{1}} \\
t_{2}=\frac{L p_{2}}{k v_{2}} \\
t_{3}=\frac{L\left(1-p_{1}-p_{2}\right)}{k v_{3}} \\
L\left[p_{1} v_{2} v_{3}+p_{2} V_{1} v_{3}+\left(1-p_{1}-p_{2}\right) v_{1} v_{2}\right] \\
k v_{1} v_{2} v_{3}
\end{gathered}
$$




$$
v=\frac{L}{T}=\frac{k v_{1} v_{2} v_{3}}{p_{1} v_{2} v_{3}+p_{2} v_{1} v_{3}+\left(1-p_{1}-p_{2}\right) v_{1} v_{2}}
$$

As for the refractive index $n$,

$$
n=\frac{c}{v}=\frac{c\left[p_{1} v_{2} v_{3}+p_{2} v_{1} v_{3}+\left(1-p_{1}-p_{2}\right) v_{1} v_{2}\right]}{k v_{1} v_{2} v_{3}}=p_{1} \frac{\left(c v_{3}-c v_{1}\right)}{k v_{1} v_{3}}+p_{2} \frac{\left(c v_{3}-c v_{2}\right)}{k v_{2} v_{3}}+\frac{c}{k v_{3}}
$$

As shown in Eq.(14), the refractive index varies linearly with molar concentrations in the ternary system solution. The solution's refractive index change rate, which is a result of changes in the molar concentrations of the two solutes, depends on the solution properties: Namely, the speed of light in the solution $(\mathrm{kmol} / \mathrm{L})$.

\section{Experimental Verification}

\section{Experimental Methods and Data Collection}

The refractive index of the ternary system solution containing two typical solutes $\left(\mathrm{CuSO}_{4}-\mathrm{NaCl}-\mathrm{H}_{2} \mathrm{O}\right)$ was measured to verify the model discussed above. There were six solution groups $\left(\mathrm{CuSO}_{4}-\mathrm{NaCl}-\mathrm{H}_{2} \mathrm{O}\right)$ with 36 kinds of molar concentrations under analysis. $\mathrm{TheCuSO}_{4}$ and $\mathrm{NaCl}$ samples were analytically pure $(\geq 99.9 \%)$. $\mathrm{CuSO}_{4}$ and $\mathrm{NaCl}$ molar concentrations varied from $0-0.5 \mathrm{~mol} / \mathrm{L}$ at change rate of $0.1 \mathrm{~mol} / \mathrm{L}$. The temperature was $18.5^{\circ} \mathrm{C}\left( \pm 0.5^{\circ} \mathrm{C}\right)$, and a $589 \mathrm{~nm}$ sodium light served as the light source. A $2 \mathrm{~W}$ Abbe refracttometer (Shanghai Optical Instrument Factory) was used to measure the refractive index of the six solution groups $\left(\mathrm{CuSO}_{4}-\mathrm{NaCl}-\mathrm{H}_{2} \mathrm{O}\right)$ with 36 molar concentrations. The measurement results are shown in Table 1.

\begin{tabular}{|c|c|c|c|c|c|c|c|}
\hline & & \multicolumn{6}{|c|}{$\mathrm{NaCl}(\mathrm{mol} / \mathrm{L})$} \\
\hline & & 0 & 0.1 & 0.2 & 0.3 & 0.4 & 0.5 \\
\hline \multirow{6}{*}{ 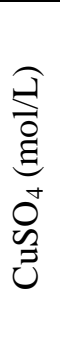 } & 0 & 1.3342 & 1.3352 & 1.3361 & 1.3371 & 1.3381 & 1.3391 \\
\hline & 0.1 & 1.3369 & 1.3379 & 1.3390 & 1.3399 & 1.3409 & 1.3419 \\
\hline & 0.2 & 1.3402 & 1.3411 & 1.3422 & 1.3432 & 1.3442 & 1.3451 \\
\hline & 0.3 & 1.3430 & 1.3440 & 1.3449 & 1.3460 & 1.3469 & 1.3479 \\
\hline & 0.4 & 1.3460 & 1.3470 & 1.3479 & 1.3489 & 1.3498 & 1.3509 \\
\hline & 0.5 & 1.3487 & 1.3498 & 1.3509 & 1.3519 & 1.3529 & 1.3538 \\
\hline
\end{tabular}

Table 1 Refractive indexes of $\mathrm{CuSO}_{4}-\mathrm{NaCl}-\mathrm{H}_{2} \mathrm{O}$ with different molar concentrations

\section{Data Analysis}

The relationship between the mixed liquid refractive index and theCuSO${ }_{4}$ and $\mathrm{NaCl}$ molar concentrations determined by processing the above data via linear regression is illustrated in Fig. 2. Coefficient $R^{2}$ above 0.999 was considered to indicate a close linear fit. Regardless of changes inCuSO$_{4}$ or $\mathrm{NaCl}$ molar concentration, the refractive index of $\mathrm{CuSO}_{4}-\mathrm{NaCl}-\mathrm{H}_{2} \mathrm{O}$ solution varied linearly. In addition, these changes had no significant effect on the refractive index in accordance with the proposed model.

TheCuSO $4-\mathrm{NaCl}-\mathrm{H}_{2} \mathrm{O}$ solution contained $\mathrm{NaCl}$ solution $(1 \mathrm{~mol} / \mathrm{L})$ and $\mathrm{CuSO}_{4}$ solution (1 $\mathrm{mol} / \mathrm{L}$ ); liquids 1and 2were selected with known molar concentrations and a known refractive index. The actual refractive index of the $\mathrm{NaCl}$ solution $(1 \mathrm{~mol} / \mathrm{L})$, CuSO4 solution $(1 \mathrm{~mol} / \mathrm{L})$, and deionized water comprised the benchmark. The refractive indexes of the ternary system solutions with different molar concentrations were calculated accordingly. The calculated and experimental results were in accordance, with a mean relative error of $5.56 \times 10^{-5}$.Thisconfirms the feasibility and effectiveness of the proposed equivalent analysis and mathematical modeling technique for ternary system solution refractive indexes. 


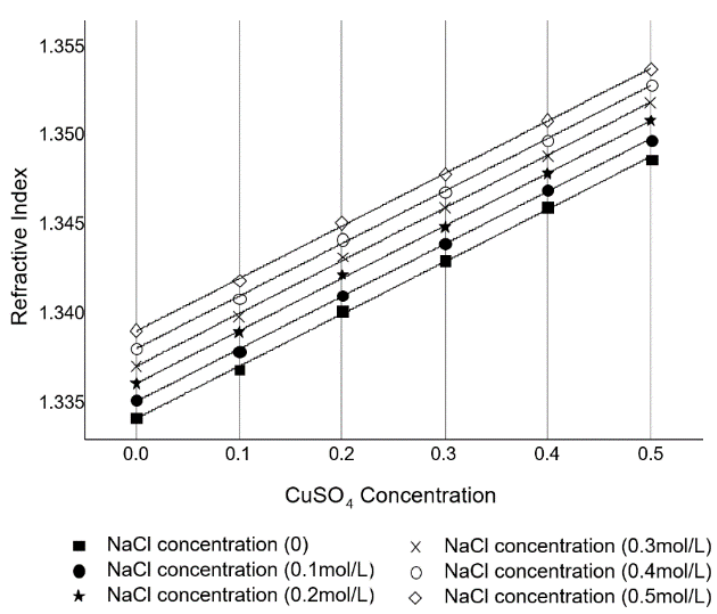

Fig. 2 Relationship between refractive index of $\mathrm{CuSO}_{4}-\mathrm{NaCl}-\mathrm{H}_{2} \mathrm{O}$ and molar concentration of $\mathrm{CuSO}_{4}$

\section{Refractive Index Multi component Solution Analysis}

The relationship between the $N$-ary solution's refractive index and each solute's molar concentration was analyzed based on assessment of the ternary system solution's refractive index and subsequent experimental verification. The $N$-ary solution was regarded as a combination of $N$ kinds of liquids with constant molar concentrations and refractive indexes. Liquid 1, liquid 2, and liquid $(N-1)$ were considered to be solutions with constant molar concentrations and refractive indexes which combine intosolutes $X_{1}$, and $X_{2}$, respectively. The molar concentration of liquid $m(m$ $\in[1, N-1])$ is $\mathrm{kmol} / \mathrm{L}$. Liquid $N$ is a solvent with constant molar concentration and refractive index (the remaining solvent after removing what was combined in liquid 1, liquid 2, and liquid $(N-1)$ ).

Light speed in a vacuum is $c$, light speed in the solution is $v$, light speed in liquid $m$ is $v_{\mathrm{m}}$, and the solute's molar concentration $X_{\mathrm{m}}$ is $p_{m} \mathrm{~mol} / \mathrm{L}$. For a unit volume in $N$-ary solution, it is assumed that the liquid volume with known refractive index and molar concentration is $V_{\mathrm{m}}$ and the remaining solvent volume is $V_{\mathrm{N}}$. The distance of light passing through the solution is Land the total time is $T$; the distance of light passing through the liquid $m$ is $L_{\mathrm{m}}$ and the time is $t_{\mathrm{m}}$.

The quality of solute $X_{\mathrm{m}}$ in the solution is the same as that in liquid $m$, thus:

$$
\begin{gathered}
p_{m} V=k V_{m} \\
p_{m}=k \frac{V_{m}}{V} \\
L_{m}=\frac{L p_{m}}{k} \\
L_{N}=\frac{L\left(1-\sum_{i=1}^{N-1} p_{i}\right)}{k} \\
t_{m}=\frac{L p_{m}}{k v_{m}} \\
t_{N}=\frac{L\left(1-\sum_{i=1}^{N-1} p_{i}\right)}{k v_{N}} \\
T=\sum_{i=1}^{N-1} \frac{L p_{i}}{k v_{i}}+\frac{L\left(1-\sum_{i=1}^{N-1} p_{i}\right)}{k v_{N}}
\end{gathered}
$$




$$
n=\frac{c}{v}=\frac{T c}{L}=\sum_{i=1}^{N-1} \frac{c p_{i}}{k v_{i}}+\frac{c\left(1-\sum_{i=1}^{N-1} p_{i}\right)}{k v_{N}}=\sum_{i=1}^{N-1} p_{i} \frac{c\left(v_{N}-v_{i}\right)}{k v_{i} v_{N}}+\frac{c}{k v_{N}}
$$

The refractive index is linearly related to the molar concentrations of $(N-1)$ solutes for $N$-ary solution. The molar concentration change rate is dependent on the light speeds in $(N-1)$ liquids $v_{m}(m$ $\in[1, N-1])$.

The above equation can also be writtenas follows:

$$
n=\sum_{i=1}^{N-1} \frac{c p_{i}}{k v_{i}}=\sum_{i=1}^{N} \frac{c V_{i}}{v_{i} V}=\sum_{i=1}^{N} \frac{v_{i} V_{i}}{v_{i} V}+\sum_{i=1}^{N} \frac{\left(c-v_{i}\right) V_{i}}{v_{i} V}=1+\sum_{i=1}^{N} p_{i} \frac{\left(c-v_{i}\right)}{k v_{i}}
$$

where $c$ is light speed in a vacuum, $p_{\mathrm{i}}$ is the solute's molar concentration $X_{\mathrm{m}}, v_{\mathrm{i}}$ is light speed in the solute with constant molar concentration of $\mathrm{kmol} / \mathrm{L}$ and constant refractive index, $v_{\mathrm{N}}$ is light speed in the solution, and $k$ is the liquid molar concentration with constant molar concentration and refractive index.

\section{Conclusion}

In this study, a multi-component solution was modeled and deduced according to the time necessary for monochromatic light to pass through the medium. The multi component system refractive index was found to be linearly related to each solute's molar concentration. A detailed correlation model of the multi-component solution refractive index was proposed, and the model was verified by a ternary system solution experiment. It is important to note that the model was established without considering the interaction between solute ions, and thus would not work well for a solution in which volume is significantly reduced after dissolution (e.g., water and ethanol). Further research is needed to make the necessary volume change correction.

\section{References}

[1] Jim'enezRiob'oo R J, Philipp M, Ramos M A et al. Concentration and Temperature Dependence of the Refractive Index of Ethanol-Water Mixtures: Influence of Intermolecular Interactions[J]. The European Physical Journal E. 2009,30(10):19-26.

[2] Yahya M, Saghir M Z. Prediction and Experimental Measurement of Refractive Index in Ternary Hydrocarbon Mixtures[J]. Journal of Chemical \& Engineering Data, 2015, 60 (8): 2329-2342.

[3] Ma Xiaochun, Dong Junliang, Liang Fang et al. A Fiber Optic Salinity Measurement Method Based on the Fresnel Principle[J]. Laser Technology, 2010, 34(3):313-315.

[4] Zhao Dewei. A Novel Fast Detecting Device for Beer Quality[J]. Global Alcohol Industry Information. 2015(1):47-48.

[5] Glover F A, Goulden J D S, Relationship Between Refractive Index and Concentration of Solutions[J]. Nature, 1963, 200(4912): 1165-1166.

[6] Zhang Zhiwei, Yin Weifeng, Wen Tingdun et al. Study on Relational Expression of Solution Concentration and Its Refractive Index[J]. Journal of North University of China (Natural Science Edition), 2009, 30(3): 281-285.

[7] Zhu Min, XinDuqiang, XieYanlei et al. A method to calculate the refractive index of ternary inorganic solution[J]. Journal of Beijing Normal University (Natural Science), 2006, 42(2):174-176.

[8] Zhang Tao. Refraction of light in medium[J]. Science in China (Physics, Mechanics \& Astronomy), 2007, 37(2): 137-145. 